\title{
Birefringence patterns in diamond
}

\author{
SIR C V RAMAN and G R RENDALL \\ Department of Physics, Indian Institute of Science, Bangalore
}

Received May 11, 1944

\section{Introduction}

Mineralogists have long been familiar with the fact that diamonds exhibit a very varied behaviour when examined under the polarising microscope. Sinor (1930) made extensive observations on the diamonds mined in the Panna State and found that very clear crystals in which all the faces were symmetrically developed showed very little or no double refraction. On the other hand, crystals containing flaws and inclusions, distorted crystals, and brownish tinted crystals showed bands and stripes of colour between crossed nicols. Since perfection of external form and of optical quality are both indicative of a homogeneity of structure, the specimens exhibiting them should naturally also show the optical isotropy characteristic of a cubic crystal. Per contra, it is not surprising that diamonds having an irregular form or visible internal flaws or inclusions should exhibit birefringence. Even a small difference in crystal orientation in the different parts of a diamond which has solidified under unsatisfactory conditions would result in enormous stresses and strains being set up which would reveal themselves in the polariscope. Such birefringence would necessarily be irregular. The variations of crystal orientation within the diamond giving rise to it should be evident on an $\mathrm{X}$ ray examination, e.g., in a Laue photograph, the spots in the pattern being distorted or drawn out into streaks or even appearing multiplied in number.

Birefringence of a wholly different kind which may be described as structural and not as accidental, is also exhibited by many specimens of diamond. Such birefringence is distinguished by the geometric character of the figures observed in the polariscope, as also by the relationship of the figures to the crystal architecture and symmetry. Birefringence of this kind in diamond and its interpretation forms the subject of the present paper. Somewhat analogous patterns are exhibited by crystal plates of isomorphous mixtures of substances crystallising in the cubic system, e.g., the nitrates of barium, strontium and solids, and especially by crystal plates of the mixed alums when examined between crossed nicols (Liebisch 1896). As is shown in the introductory paper of this symposium, crystallographic considerations as well as spectroscopic evidence 
compel us to admit the possibility of four different structures for diamond, two with tetrahedral and two others with octahedral symmetry, which may coexist in one and the same specimen. This fact opens the way to an understanding of the geometric birefringence patterns observed in diamond, as also of other properties which vary with its structure, e.g., its luminescence, its transparency in the visible, ultra-violet and infra-red regions of the spectrum, its reflecting power for X-rays and its photoconductivity, all of which stand in close relation to each other.

\section{Crystals in their natural form}

Observations with diamonds in their natural state placed between crossed nicols are of interest in view of the fact that the absence of birefringence is a very delicate test of crystal perfection. Consider for instance, an octahedral diamond of 20 carats which would be about a centimeter thick and the optical path through which would be some 50,000 wavelengths of visible light. A difference of one part in a million in the refractive indices for vibrations in different directions would produce an easily observable restoration of light between crossed nicols, while a difference of one part in a hundred thousand would give polarisation colours. It will be evident that the test is an extraordinarily sensitive one for crystal perfection, especially where large diamonds are concerned.

Owing to the high refractivity of diamond, such observations are necessarily be confined in any one setting of the crystal to small portions of it bounded by parallel faces. These regions would be further restricted if, as is often the case, the surfaces of the crystal are curved. The difficulties mentioned are least serious in the case of large regular crystals with nearly plane faces and most serious for small crystals having highly curved surfaces and with irregular diamonds. They may, however, be minimised by immersing the crystal in a cell containing a transparent liquid of sufficiently high refractive index.

The optical behaviour of numerous diamonds, including some of large size and in particular, the Maharajah's necklace of 52 octahedral crystals was studied by Sir C V Raman and Dr P G N Nayar during their visit to Panna State in July 1942. More recently at Bangalore, 42 Panna crystals of various sizes and qualities have been critically examined for birefringence when immersed in liquid monobromnaphthalene. The conclusions indicated by these studies are as follows:

(a) Diamonds which are of the blue luminescent and ultra-violet opaque type are optically isotropic, their birefringence, if any, being less than 1 part in 100,000. This is subject to the proviso that the crystal is of perfect form, free from flaws and inclusions, and colourless.

(b) Small diamonds of the kind stated above may be optically isotropic even if the crystal form is not absolutely regular. 
(c) Other diamonds usually exhibit marked birefringence of which there are two kinds, appearing each by itself or together, viz., birefringence which is wholly irregular, and birefringence which is related to the crystal architecture and exhibits a geometric character.

(d) While birefringence of the latter kind may appear in blue-luminescent diamonds, it is an invariable feature in the yellow-luminescing ones.

\section{Observations with polished cleavage plates}

There are notable advantages in the study of the birefringence patterns in diamond gained by the use of polished cleavage plates, such as are readily obtainable. As these plates are parallel to the octahedral planes (more rarely to the dodecahedral planes) in the crystal, the relationship of the pattern seen in the polariscope to the crystal architecture is immediately apparent. Moreover, the pattern over the whole area of the plate is visible and can be photographed at the same time, while the disturbing effects due to oblique reflection and refraction which are so troublesome in working with crystals in their natural form do not arise at all. There is also another notable gain in the use of cleavage plates. The act of cleavage releases the material in the plate from stresses having their origin in faults or irregularities elsewhere in the original crystal from which it is split off. The accidental or irregular birefringence due to those stresses is thereby eliminated and the real optical character of the material in the plate made accessible to observation.

A beautiful illustration of the foregoing remarks is furnished by the case of the cleavage plates D36 and D45, the birefringence patterns of which are reproduced in juxtaposition in figure 4 of plate IV. These two diamonds originally formed a single plate, the whole area of which exhibited a strong restoration of light between crossed nicols. This had its origin in certain irregularities located in the lower part included in D36 and which are clearly seen in the pattern of the latter. When D45 broke off, the stresses in it were released with the result that the birefringence in it has disappeared, while that in D36 remains undiminished (possibly even a little enhanced).

The birefringence patterns as seen between crossed polaroids of several cleavage plates are reproduced as figures $1,2,3$ and 4 in plates I to IV accompanying this paper. These were chosen from amongst a large number so as to be generally representative of the whole and at the same time to illustrate points of special interest. The significance of these patterns becomes evident when they are carefully compared with the luminescence patterns, the ultra-violet transparency patterns and X-ray topographs of the same diamonds appearing with the papers immediately following the present one in the symposium. 


\section{Description of the patterns}

The diamonds D221 (figure 1) and D45 (figure 4) are opaque to the $2536 \AA$ radiations of the quartz mercury arc lamp and are both feebly blue-luminescent. It will be noticed from the figures that they give no sensible restoration of light between the crossed polaroids, thereby confirming the essentially isotropic character of diamonds of this class already made evident from the study of the best Panna crystals. The statement made by Robertson, Fox and Martin (1934) that type I or ultra-violet opaque diamonds are optically anisotropic is thus clearly not justified.

D174, D178 and D179 (figure 1) are three diamonds which exhibit rather striking patterns of geometric type. These diamonds are, over the greater part of their area, of the blue-fluorescent and ultra-violet opaque type. That they are optically isotropic in the same regions is evident from the fact that nearly the whole area of D178, the central region of D174 and the major part of the area of D179 remain quite dark as seen at all settings between crossed polaroids. The symmetric patterns shown rather feebly in D178 and more strongly in D174 and D179 consist of bands running parallel to the octahedral planes in the crystal. The region of the diamond which appears as a particularly bright line in the birefringence pattern of D179 appears as a dark line in its luminescence pattern and as a line of diminished opacity in its ultra-violet transparency pattern, thereby clearly showing that it is an intrusive layer of diamond having different properties from the rest of the material in the plate. The same result is indicated by the X-ray topographs of D174 and D179 obtained by Mr G N Ramachandran (1944), where the intruding layers reveal themselves by the increased intensity of $\mathrm{X}$-ray reflection at precisely the same regions in the plates. D181 and D38 illustrated in figure 1 are also blue-luminescent diamonds. The strong birefringence which they exhibit has essentially the same explanation, viz., the intrusion into the blue-luminescent diamond of thick layers of non-luminescent diamond. This is clearly shown by the X-ray topographs which exhibit a perfect correspondence with the birefringence patterns.

The appearance between crossed polaroids of D206, D207, D208, D209 and of D39 and D57 is illustrated in figures 2, 3 and 4. All these six diamonds are of the ultra-violet transparent and non-fluorescent class, and it will be seen that the birefringence which they exhibit is of a highly characteristic type, viz., sets of parallel dark and bright streaks running through the crystal in various directions. The spacing of these streaks is extremely variable, and indeed, both coarsely and finely-spaced streaks may often be seen at the same time in any particular specimen. A special remark is necessary regarding D39, which at first sight seems different in its behaviour from the others. In this diamond, an irregular birefringence is present, due to some obvious imperfections in the crystal which are also revealed by $\mathrm{X}$-ray examination. This obscures the characteristic streaky birefringence of diamonds of this class over a greater part of its area. Careful 
examination under higher magnification, however, reveals the presence of the latter, and especially clearly when the irregular birefringence is eliminated in a particular area by an appropriate setting of the plate between the crossed polaroids. The patterns of D57 appearing in figure 4 have been reproduced under rather high magnification in order to exhibit the characteristic criss-cross streakiness of the field more clearly.

The case of D39 (and also of some other diamonds in the collection) shows clearly that diamonds of the ultra-violet transparent class may exhibit irregular birefringence. The statement made by Robertson, Fox and Martin in their paper that diamonds of this class are optically isotropic is therefore not justified. While all diamonds may exhibit irregular birefringence if they have imperfections, perfect crystals of the blue-fluorescent ultra-violet opaque type are, as we have seen, essentially isotropic and free from birefringence, while diamonds of the nonfluorescent ultra-violet transparent type have a characteristic structural birefringence of the kind illustrated in figures 2, 3 and 4 in the plates. The situation is thus actually the reverse of that stated to exist by Robertson, Fox and Martin.

The remaining patterns illustrated in the plates accompanying the paper fall into two groups. Some of them are essentially of the same nature as the patterns of the six ultra-violet transparent diamonds considered above. The diamonds D199 and D202 are of this kind. They exhibit a yellow fluorescence. They are not fully ultra-violet transparent, but as has been shown in the paper by Sunanda Bai (1944), if adequate exposures are given, the recorded absorption spectra of these diamonds extend to the same limit as that of the fully ultra-violet transparent diamonds. It is therefore scarcely surprising that the birefringence patterns are also of the same nature for the two sets of diamonds. The other two plates whose patterns have been reproduced, viz., D48 and D235, are typical mixed diamonds, showing in different parts of their area all the three kinds of behaviour in respect of luminescence, viz., blue-luminescence, non-luminescence and yellowluminescence, and the corresponding three different behaviours in their ultraviolet absorption, viz., opacity, perfect transparency and partial transparency. The respective regions in the areas of the plate can be distinguished in the luminescence and ultra-violet transparency patterns, while the X-ray topographs show clearly the intrusion into each other of the different types of diamond. The corresponding variations in the nature of the birefringence in different areas of the plate can also be readily made out in figures 2 and 4 .

\section{The origin of structural birefringence}

The origin of irregular birefringence has already been considered and pointed out earlier in the paper, viz., accidental imperfections of the crystal structure. The appearance of such imperfections is scarcely surprising when the structure of 
diamond and the nature of the atomic forces in it are considered. The binding forces acting directly between neighbouring atoms of carbon are the strongest of these and are sufficient to secure the coherence of all the atoms in a specimen even when the circumstances of formation of the crystal are such that complete uniformity of the inter-atomic distances and especially of the valence angles throughout its volume is not possible. Atomic equilibrium can then only be secured by the existence of a system of macroscopic stresses and strains in the solid.

The origin of the kind of birefringence with which we are principally concerned in this paper is, however, of an altogether different nature. As we have seen, it arises from the co-existence in the same specimen and interpenetration into each other of kinds of diamond having different physical properties. Considering, first, the diamonds of the blue-luminescent ultra-violet opaque class, these have tetrahedral symmetry, the two variants $T d$ I and $T d$ II having this symmetry being co-existent and interpenetrating to varying extents. The absence of birefringence in such diamonds indicates that the crystal spacings of these two structures are perfectly identical and that they can therefore fit into each other without any stresses or strains arising. Indeed, the relationship between neighbouring carbon atoms in Td I and Td II is physically the same, but geometrically different. Hence, the identity of crystal spacing and the absence of any birefringence in diamonds of this class is fully to be expected. Per contra, the appearance of a characteristic streaky or lamellar birefringence in the nonluminescent ultra-violet transparent diamonds indicates that the $\mathrm{Oh} I$ and $\mathrm{Oh}$ II structures of which these diamonds consist, and the co-existence and interpenetration of which gives rise to their lamellar structure, are not physically identical. It is evident that even a small difference in the crystal spacing of the two interpenetrating forms would give rise to a streaky or lamellar birefringence, and that it would also give rise to marked inhomogeneities giving a greatly increased intensity of X-ray reflection.

The explanation of structural birefringence in diamond indicated above is confirmed in a striking manner by the existence of observable variations of crystal spacing in diamonds of the ultra-violet transparent class. This is shown in another paper by Dr R S Krishnan (1944) appearing in this symposium. His experiments were made with the diamond D209 whose birefringence pattern is reproduced in figure 2 of the plates accompanying this paper. The variations in crystal spacing in alternate layers of the diamond were revealed by X-ray methods and found to be accompanied by periodic variations in the intensity of the X-ray reflections from these layers, indicating the existence of a close physical relationship between the two effects. The periodic variations in crystal spacing found were of the order of 5 parts per 10,000. Small though these are, they are clearly sufficient to explain the observed birefringence which is itself small and is so readily noticed only because of the delicacy of the method of observation.

$\mathrm{X}$-ray observations with specimens, such as D48 and D235, in which the 
tetrahedral and octahedral varieties of diamond appear juxtaposed in adjacent areas should similarly be capable of ascertaining the differences in crystal spacing of the Td structures from those of the Oh I and Oh II types. A knowledge of these differences would assist in a fuller elucidation of the birefringence patterns observed in such cases. In some of these patterns, e.g., those of D174 and D178 appearing in figure 1 , only the intruding layers of octahedral diamond show an appreciable restoration of light, while in others e.g., the patterns of D181 and D38, the entire diamond shows a restoration though of varying intensity, though the intrusions extend over only part of its area. It would seem that in cases of the latter kind, the stresses set up by the presence of the intruding layers extend over the entire diamond and are of sufficient magnitude to cause an appreciable birefringence to be exhibited by it.

\section{Nature and magnitude of the stresses}

Since the birefringence patterns are photoelastic effects due to the variations of the crystal spacing in diamond, it follows that the axes of birefringence should be related to the orientation of the layers in the crystal in a determinate way. In particular, when there is only one set of laminations in the crystal, the axes of birefringence should be parallel and perpendicular respectively to the laminations. This is readily tested by placing the diamond between crossed polaroids and observing the changes in the pattern as the diamond is rotated in its own plane. As is to be expected, it is found that the bands in the pattern appear most intense when they run at an angle of $45^{\circ}$ with the axes of the polaroids and vanish when they are set parallel or perpendicular to them.

In more complicated cases when there are two or more sets of parallel laminations running in different directions and intersecting each other, both the nature of the pattern and the axes of birefringence would be determined by their joint effect, and not by any one of them separately. A striking illustration of this is furnished by D209, the birefringence patterns of which taken at two different settings of the diamonds are reproduced in figure 2 . It will be noticed that in one setting, the pattern is very bright and shows a rectangular patchwork of dark and bright lines, while in the other setting, it is of much smaller intensity and of irregular character. It is found that the pattern is most intense when the bands in the birefringence pattern are inclined at $45^{\circ}$ to the axes of the crossed polaroids, and least intense when they are parallel and perpendicular respectively to these axes. It may be remarked that the X-ray topograph of D209 shows the presence of two sets of strongly reflecting layers of diamond which are inclined to each other at an angle of $60^{\circ}$, while the birefringence pattern, on the other hand, shows a rectangular pattern of bright and dark bands. This difference is, however, entirely to be expected in view of the remarks made above. Indeed, the resemblances as well as the differences noticed between the birefringence pattern of this diamond and 
its X-ray topograph form a striking confirmation of the explanations put forward of the origin of the former pattern.

Quantitative measures of the birefringence are obviously desirable to enable a more complete account of the subject to be given. A knowledge of the elastic-optic constants of diamond, the orientation and thickness of the intruding layers, and of their crystal spacings, should enable the expected birefringence to be computed and compared with the observed values. Such an investigation should be well worth undertaking. It should be remarked also that the birefringence patterns reveal only the differences in the refractive index for vibrations along the principal axes of stress. Observations of interference patterns may conceivably reveal the absolute variations of the refractive index, though these would naturally be very small. A knowledge of them would be necessary for a complete evaluation of the stress system of pressures and shears present in the diamond.

\section{Summary}

Birefringence in diamond may be either irregular or of geometric character. In the former case, it is due to structural imperfections, but its magnitude is negligible in well-formed perfect crystals. Geometric or structural birefringence manifests itself in regular patterns related to the symmetry of the crystal. It arises from the coexistence in the crystal of structures with different properties and crystal spacings. Extensive studies prove that diamond of the blue-luminescent ultra-violet opaque type is isotropic, while diamond of the non-luminescent ultra-violet transparent type invariably shows a structural birefringence. These facts find a natural explanation when the relationships existing between the four possible structuretypes in diamond namely $\mathrm{Td} \mathrm{I}, \mathrm{Td} \mathrm{II}, \mathrm{Oh} \mathrm{I}$ and $\mathrm{Oh} \mathrm{II}$ are considered. A difference in the crystal spacings of the Oh I and Oh II structures present in the ultra-violet transparent diamonds is proved by X-ray studies and is the origin of the streaky or laminar birefringence exhibited by such diamonds. Birefringence patterns may also arise from the intrusion of the Oh structure into Td diamonds. This is fully confirmed by the variations of luminescence, ultra-violet transparency, and of Xray reflection intensities over the area of the specimen exhibited by such diamonds.

\section{References}

Krishnan R S, Proc. Indian Acad. Sci.A19 (1944)

Liebisch T, Physikalische Krystallographie Veit, Leipzig 325 (1896)

Ramachandran G N, Proc. Indian Acad. Sci. A19 (1944)

Robertson, Fox and Martin, Philos. Trans. R. Soc. A232 463 (1934)

Sinor The diamond mines of Panna (1930).

Sunanda Bai K, Proc. Indian Acad. Sci. A19 253 (1944) 


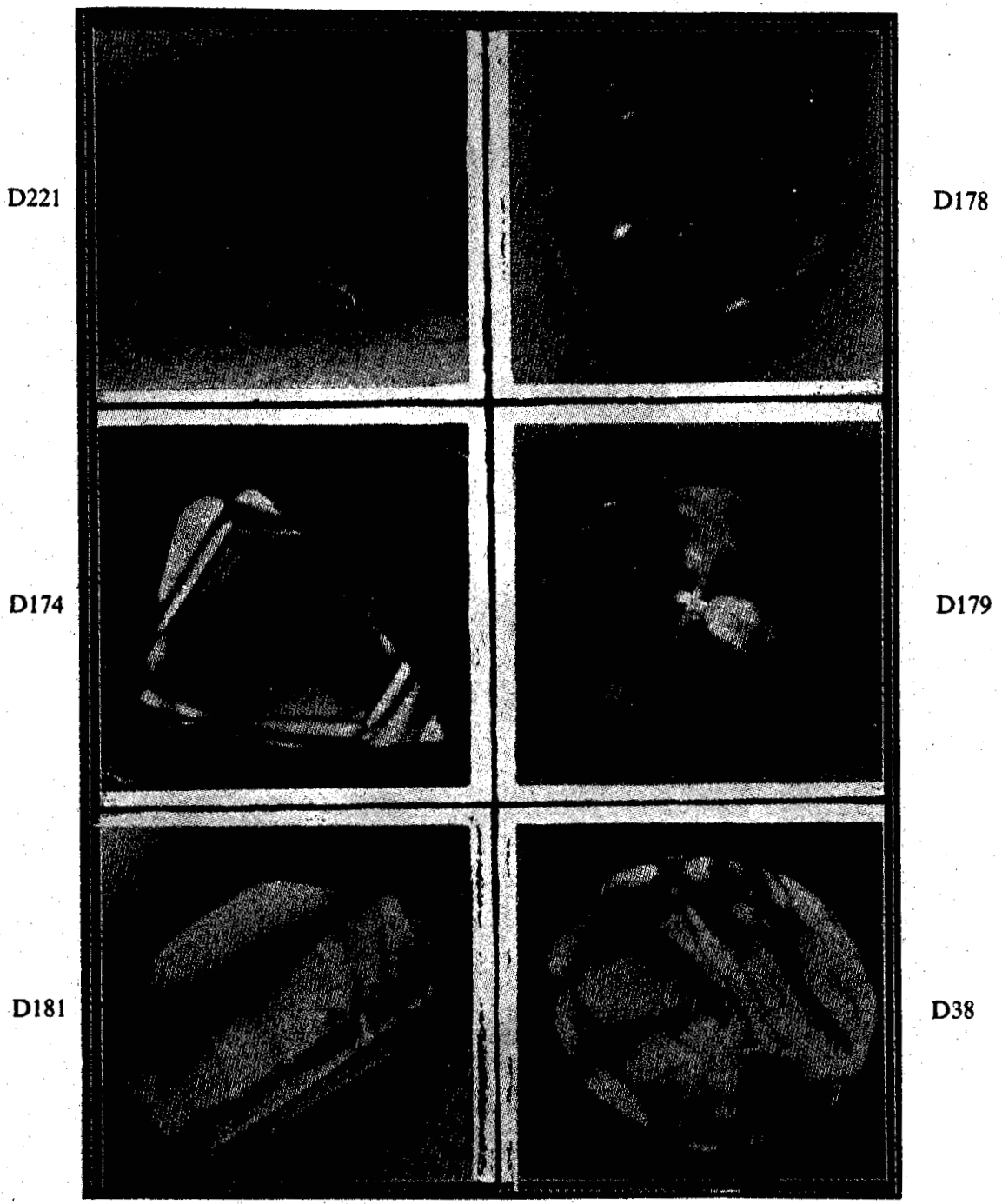

Figure 1. Birefringence patterns in blue-luminescent diamonds.

Plate I 


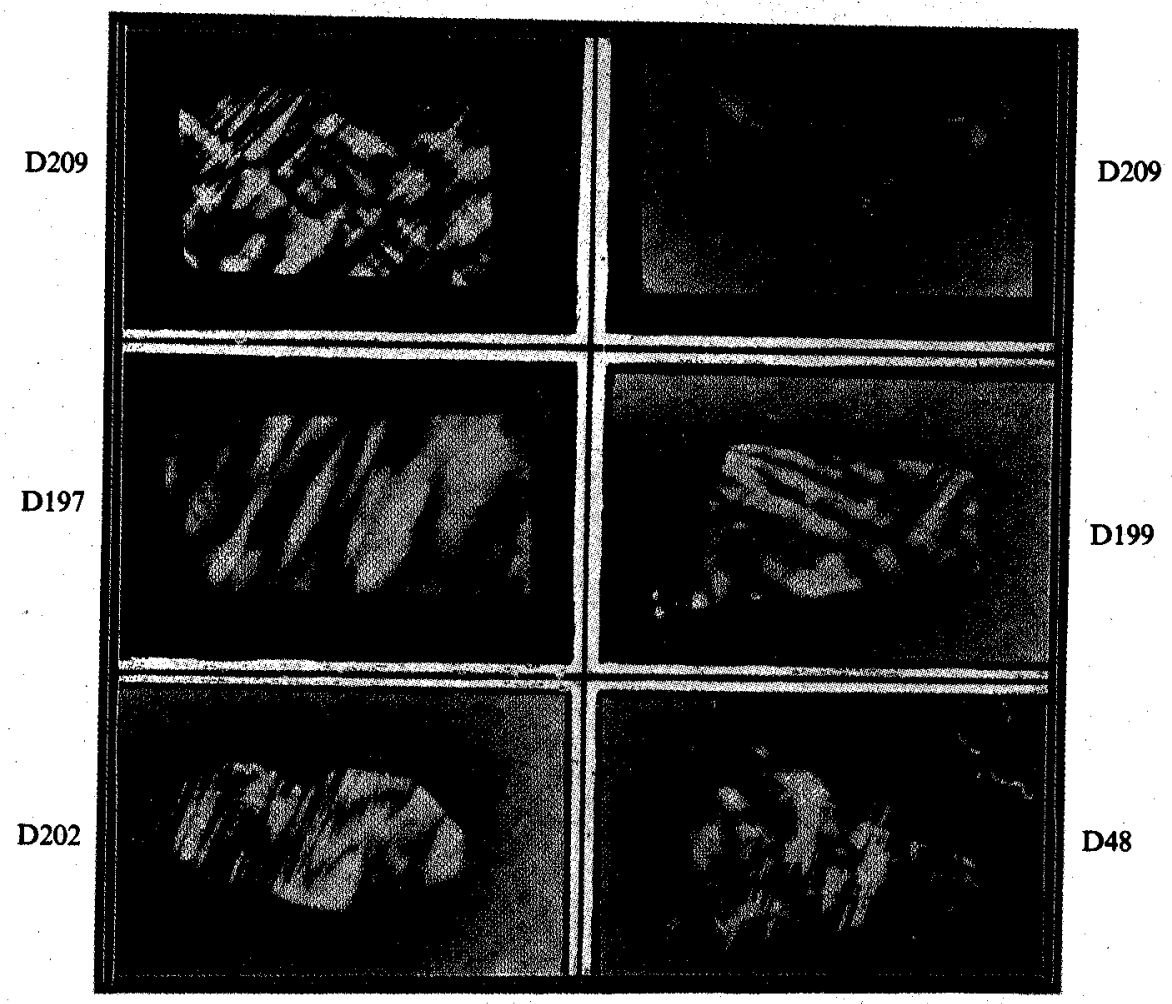

Figure 2. Streaky birefringence in diamonds.

Plate II 


\section{$\gamma$}

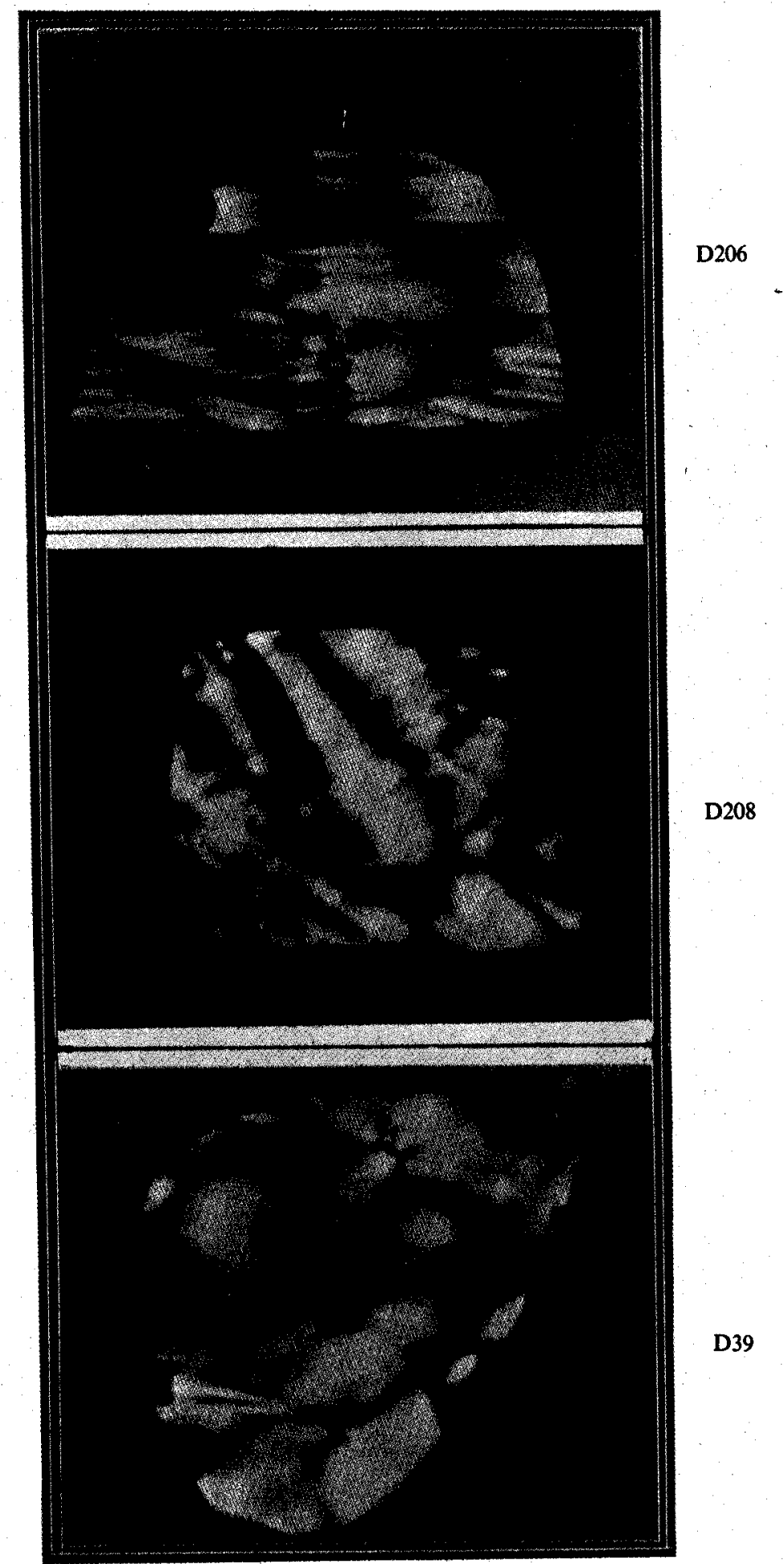

Figure 3. Birefringence in non-luminescent diamonds.

Plate III 


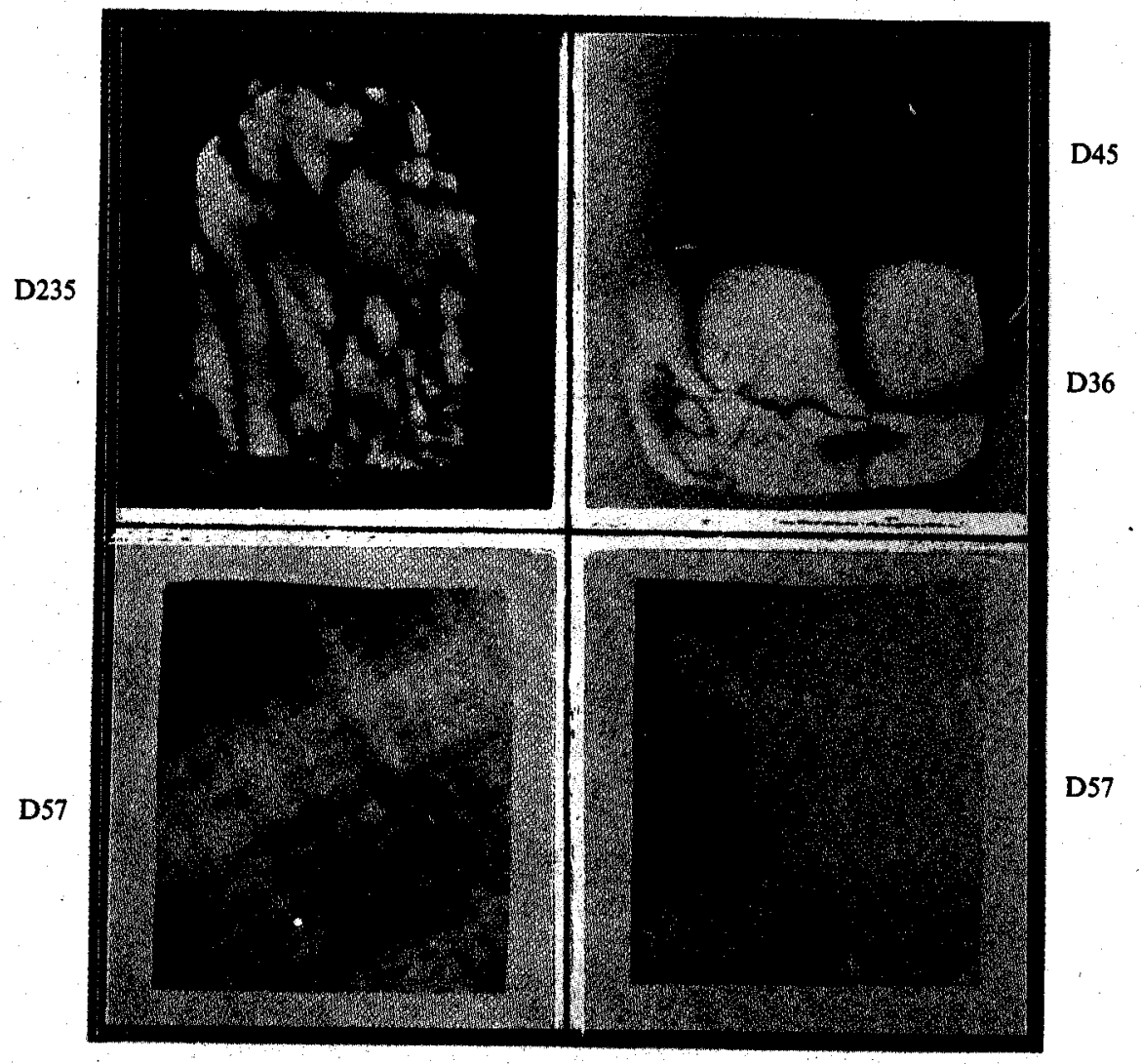

Figure 4. Birefringence in diamonds.

Plate IV 